\title{
Extracranial vertebral artery dissection following tonic clonic seizure
}

\author{
C A Young, D W Chadwick, P R D Humphrey
}

\begin{abstract}
A 34 year old woman developed cerebral infarction, following a tonic-clonic seizure. A vertebral artery dissection was demonstrated and may have been caused by arterial trauma during the seizure. This cause of morbidity following convulsive seizures may have been overlooked in the past and needs to be recognised in view of the potential benefits of anticoagulation.
\end{abstract}

Extracranial dissection of carotid or vertebral arteries is an increasingly recognised cause of stroke in young people. Dissection of the vertebral arteries has most frequently been reported in association with abnormal neck movements, such as may occur with chiropractic manipulation, ${ }^{1}$ yoga, ${ }^{2}$ gymnastics, ${ }^{3}$ falls or road traffic accidents. ${ }^{4}$ We report a case of vertebral artery dissection following a tonic clonic seizure.

\section{Case report}

A 34 year old woman had a history of juvenile myoclonic epilepsy well controlled by sodium valproate and had had a single post partum tonic-clonic seizure seven years previously. She had a nocturnal tonic clonic fit witnessed by her husband. The following morning she complained of headache and became disorientated and unsteady. She was admitted to hospital where the examination was normal. The following day she complained of unilateral occipital headache and paraesthesia of the left face and left hand. The next day she was dyspraxic and had a left hononymous hemianopia, mild left pyramidal signs, left somatosensory inattention, prosopagnosia, dressing apraxia and visual agnosia. CT brain scan showed a small area of infarction with a low density zone in the right temporoparietal region with peripheral enhancement after contrast.

Ultrasound Doppler scanning showed decreased flow in the left vertebral artery. Cerebral angiography showed a marked irregular extracranial narrowing of the left vertebral artery from $\mathrm{C} 1-\mathrm{C} 6$ (figure). The right vertebral and carotid arteries were normal. The patient was anticoagulated, continued on sodium valproate and made a good recovery over three months. Anticoagulation was discontinued after six months.

\section{Discussion}

Dissections are an important cause of stroke in young people. In a retrospective, hospitalbased series of 100 patients under 40 with cerebral infarction, two had carotid dissections and two had vertebral dissections following forcible head movement. ${ }^{5}$

The vertebral arteries seem to be particularly susceptible to dissection following abnormal neck movements. A review of 52 cases, average age 37 years, showed some preceding neck torsion, such as chiropractic manipulation, in 41 patients $(80 \%) .^{1}$ It is suggested that rotation and/or extension of the head compresses and stretches the vertebral arteries which are vulnerable because of their course and anatomical relationship with the sixth to the first cervical vertebrae. The arteries are relatively fixed, particularly in the

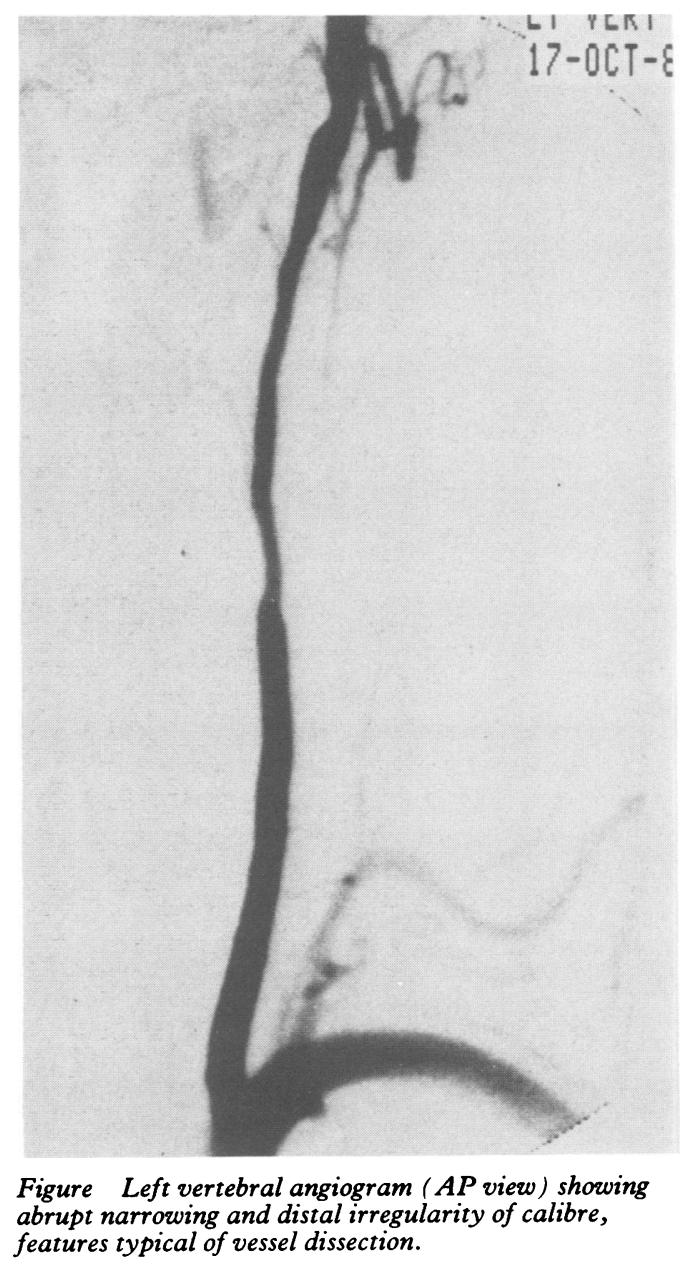


region of the atlanto-axial articulation, which is the level at which $50 \%$ of total rotation occurs.

The diagnosis of dissection depends on angiography and a rise might be anticipated in the number of cases reported with the development of improved angiographic techniques and wider recognition of the clinical and radiographic features of dissection. The age of our patient and the delayed onset and progressive development of her symptoms are common features. Sherman et $a l^{1}$ found that of the 43 cases in whom adequate clinical details were available, delayed onset of symptoms occurred in $13(30 \%)$ and progression of symptoms in $15(35 \%)$. This pattern is believed to reflect either propagation of thrombus or embolisation from the site of dissection.

Treatment is aimed at preventing secondary thrombotic and embolic complications. Mas et $a l^{6}$ report 13 cases with non-occlusive vertebral dissection, all of whom had neurological deficits of varying duration. Eleven were anticoagulated for between two and 36 months; 10 recovered without significant residual deficit. Two were treated with aspirin alone and one of these had significant residual deficit. Anticoagulation was recommended in cases where cerebrospinal fluid examination shows no evidence of subarachnoid haemorrhage. The role of anticoagulation in extracranial artery. dissection is open to debate, although most clinicians use anticoagulants if there is a significant but incomplete neurological deficit or recurrent transient ischaemic attacks. Three to 12 months is the most widely accepted length of treatment. The risk of haemorrhage into an infarct following treatment with anticoagulants is only increased in the first $\mathbf{1 0}$ days after the infarct; this risk is slight, provided the infarct is small and excessive anticoagulation (activated partial thromboplastin time $>2 x$ controls) is avoided. $^{78}$

We believe that this is the first documented case of vertebral artery dissection in association with a tonic-clonic seizure. Cases of postictal blindness have, however, been reported ${ }^{9}$ and it is possible that some of these could be due to undiagnosed vertebral artery dissection. Whilst this complication of convulsive seizures may be uncommon it should nevertheless be recognised as anticoagulation may prevent further thromboembolic episodes.

1 Sherman DG, Hart RG, Easton JD. Abrupt change in head position and cerebral infarction. Stroke 1981;12:2-6.

2 Hanus SH, Homer TD, Harter DH. Vertebral artery occlusion complicating yoga exercises. Arch Neurol occlusion comp

3 Nagler $W$. Vertebral artery obstruction by hyperextension of the neck: report of three cases. Arch Phys Med Rehab 1973;54:237-40.

4 Klein RA, Snyder RD, Schwartz HS. Lateral medullary syndrome in a child: arteriographic confirmation of vertebral artery occlusion. $J A M A$ 1976;235:940-1.

5 Hart RG, Miller VT. Cerebral infarction in young adults: practical approach. Stroke 1983;14:110-14.

6 Mas J-L, Bousser M-G, Hasboun D, Laplane D. Extracranial vertebral artery dissections: a review of 13 cases. Stroke 1987;18:1037-47.

7 Cerebral Embolism Study Group. Immediate anticoagulation of embolic stroke: a randomised trial. Stroke 1983;14:668-76.

8 Babikian VL, Kase CS, Pessin MS, Norrving B, Gorelick $\mathrm{PB}$. Intracerebral haemorrhage in stroke patients PB. Intracerebral haemorrhage in stroke patien
anticoagulated with heparin. Stroke $1989 ; 20: 1500-3$.

9 Sadeh M, Goldhammer Y, Kuritsky A. Postictal blindness in adults. JNNP 1983;46:566-9. 